\title{
Thermoregulation in Hypertensive Rats during Exercise: Effects of Physical Training
}

\author{
Luis Henrique Lobo Silame Gomes, ${ }^{1 \oplus}$ Lucas Rios Drummond, ${ }^{2 \oplus}$ Helton Oliveira Campos, ${ }^{2}{ }^{\circledR}$ Leonardo Mateus \\ Teixeira de Rezende, ${ }^{1 \oplus}$ Miguel Araújo Carneiro-Júnior, ${ }^{1 \oplus}$ Alessandro Oliveira, ${ }^{3 \oplus}$ Antônio José Natali, ${ }^{1 \oplus}$ Thales \\ Nicolau Prímola-Gomes ${ }^{10}$ \\ Universidade Federal de Viçosa (UFV), ${ }^{1}$ Viçosa, MG - Brazil \\ Universidade Federal de Minas Gerais (UFMG), ${ }^{2}$ Belo Horizonte, MG - Brazil \\ Universidade Federal de São João del-Rei, ${ }^{3}$ São João del-Rei, MG - Brazil
}

\begin{abstract}
Background: Spontaneously hypertensive rats (SHR) show deficit in thermal balance during physical exercise.

Objective: To assess the effects of low-intensity physical exercise training on thermal balance of hypertensive rats undergoing an acute exercise protocol.
\end{abstract}

Methods: Sixteen-week-old male Wistar rats and SHR were allocated into four groups: control Wistar rats (C-WIS), trained Wistar (T-WIS), control SHR (C-SHR) and trained SHR (T-SHR). Treadmill exercise training was performed for 12 weeks. Blood pressure, resting heart rate and total exercise time was measured before and after the physical exercise program. After the exercise program, a temperature sensor was implanted in the abdominal cavity, and the animals subjected to an acute exercise protocol, during which core temperature, tail skin temperature and oxygen consumption until fatigue were continuously recorded. Mechanical efficiency (ME), work, heat dissipation threshold and sensitivity were calculated. Statistical significance was set at $\mathbf{5 \%}$.

Results: Physical training and hypertension had no effect on thermal balance during physical exercise. Compared with C-WIS, the T-WIS group showed higher heat production, which was counterbalanced by higher heat dissipation. Hypertensive rats showed lower ME than normotensive rats, which was not reversed by the physical training.

Conclusion: Low-intensity physical training did not affect thermal balance in SHR subjected to acute exercise. (Arq Bras Cardiol. 2019; 112(5):534-542)

Keywords: Rats; Hypertension; Exercise/physiology; Physical Exertion; Body Temperature Changes; Fatigue.

\section{Introduction}

During exercise, elevation of core temperature (Tcore) results from an imbalance between heat production and dissipation, since heat production increases exponentially before the mechanisms of heat dissipation are activated. ${ }^{1,2}$ Hyperthermia may be a sign that individuals will reach fatigue and interrupt exercise, and hence an adequate control of the Tcore is critical for maintenance of physical performance. ${ }^{3}$

Arterial hypertension is a public health problem in the world and considered one of the main risk factors for cardiovascular diseases. ${ }^{4}$ Among the experimental models used in studies on the pathophysiology of arterial hypertension, the spontaneously hypertensive rats (SHR) is the most commonly used. Similar to humans, SHR develop progressive left ventricular hypertension in response to blood pressure elevation and to increased peripheral vascular resistance. ${ }^{5,6}$

Mailing Address: Thales Nicolau Prímola Gomes •

Universidade Federal de Viçosa - Departamento de Educação Física, Av. Ph

Rolfs, s/n. Postal Code 36570-000, Campus Universitário, Viçosa, MG - Brazil E-mail: thales.gomes@ufv.br, thalesprimola@gmail.com

Manuscript received April 23, 2018, revised manuscript August 15, 2019 , accepted August 15, 2019

DOI: $10.5935 / a b c .20190050$
In recent studies of our group, we observed that untrained SHR showed disturbances in the regulation of body temperature during acute physical exercise. During exercise, hypertensive animals showed lower heat dissipation and higher heat production, leading to marked increase in Tcore compared with normotensive animals. ${ }^{7,8}$ This was associated with lower mechanical efficiency (ME) in hypertensive animals. ${ }^{7}$

Several benefits of aerobic physical training have been demonstrated in hypertensive individuals, including reduction of blood pressure, improvement of cardiac function, and reduction in total peripheral resistance. ${ }^{9,10}$ However, the effects of low-intensity, aerobic physical training on thermal balance in hypertensive animals have not been investigated.

Thus, the present study aimed to evaluate the effects of low-intensity physical training on thermal balance of hypertensive rats subjected to an acute physical exercise protocol. We tested the hypothesis that low-intensity exercise could promote positive adaptations and reversal of the effects on the thermal balance in SHRs.

\section{Methods}

\section{Experimental animals}

Sixteen-week-old normotensive Wistar rats and SHR were randomly stratified into four groups: control Wistar rats (C-WIS, 
$\mathrm{n}=8$ ), trained Wistar (T-WIS, $\mathrm{n}=8$ ), control SHR (C-SHR, $n=8)$ and trained SHR (T-SHR, $n=8)$. Sample size was determined based on sample size calculation. ${ }^{11}$ The animals were housed in group cages in a temperature-controlled room under a 12-h light-dark cycle, and had free access to water and food. Systolic blood pressure (SBP), diastolic blood pressure (DPB) and mean blood pressure (MBP) were measured using tail plethysmography (LE5001; Panlab, Spain). Resting heart rate (RHR) was measured through the sensor placed on the tail, connected to a computer system (PowerLab 4/30; LabChart/ADInstruments, USA) before the first and 48 hours after the last session of physical training. All exercise protocols were approved by the Ethics Committee of Universidade Federal de Viçosa (Protocol \# 76/2014) and conducted according to the Helsinki declaration.

\section{Physical training protocol}

Prior to the beginning of exercise training, rats were adapted to a motorized treadmill (Insight Instruments, Brazil), five minutes/day at $5 \mathrm{~m} / \mathrm{min}$ for five days. In addition, all animals underwent an incremental exercise test (starting at $5 \mathrm{~m} / \mathrm{min}$, increasing by $3 \mathrm{~m} / \mathrm{min}$ every 3 minutes until fatigue) at the beginning of the study, at week 4 and at week 8 of training to determine total exercise time (TET) and maximum running speed (MRS). The exercise program was performed five days a week, 60 minutes/day, at 50-60\% MRS for 12 weeks, in a temperature-controlled room (approximately $22^{\circ} \mathrm{C}$ ). Both intensity and duration of exercise were gradually increased as proposed by Lavorato et al. ${ }^{12}$ Animals of the control group were handled in the same manner as the hypertensive group and underwent the same treadmill exercise program two days a week, 5 minutes/day at $5 \mathrm{~m} /$ minute. $^{12}$

\section{Experimental protocol following the physical training}

\section{Familiarization with the experimental protocol}

The animals were familiarized with the treadmill (Panlab, Harvard Apparatus, Spain) - five minutes per day, 5 degrees of inclination for three consecutive days, at $11 \mathrm{~m} / \mathrm{min}, 13 \mathrm{~m} / \mathrm{min}$ and $15 \mathrm{~m} / \mathrm{min}$ ). A thermocouple was taped to the tail of the rat and the electrical stimulation delivered at $0.4-0.6 \mathrm{~mA}{ }^{7}$ This protected the animals from having their legs wrapped around the thermocouple wire and reduced their exposure to electrical stimulation during the running test. ${ }^{13}$

\section{Temperature sensor implantation}

Immediately prior to the surgery, the animals received a prophylactic dose of antibiotic (enrofloxacin $10 \mathrm{mg} . \mathrm{kg}-1$, intramuscular) and analgesics (tramadol, $4 \mathrm{mg} \cdot \mathrm{kg}^{-1}$, subcutaneously). Anesthesia was induced with $1.5 \%$ isoflurane (BioChimico, Brazil) and 100\% oxygen (White-Martins, Brazil) at constant flow of $1 \mathrm{~L} / \mathrm{min}$. Following preparation of the incision site, a temperature sensor (G2 E-Mitter, Mini-Mitter, USA) was implanted in the abdominal cavity. ${ }^{14}$ After this procedure, the animals were housed in individual boxes and received two additional doses of tramadol in regular intervals of 8 hours.

\section{Acute physical exercise protocol}

After 48 hours of recovery from the surgery, each animal underwent to two exercise sessions at constant speed $(60 \%$ of MRS), $5^{\circ}$ slope and electrical stimulation (0.4-0.6 mA) until fatigue. Treadmill speed was $16.0 \pm 0.4 \mathrm{~m} / \mathrm{min} ; 23.0 \pm 0.7 \mathrm{~m} / \mathrm{min}$; $16.2 \pm 0.5 \mathrm{~m} / \mathrm{min} ; 19.6 \pm 0.8 \mathrm{~m} / \mathrm{min}$ for C-WIS, T-WIS, $\mathrm{C}-\mathrm{SHR}$ and T-SHR, respectively. Fatigue was defined as the point when the animals were unable to keep pace with the treadmill. The animals received electrical stimulation up to ten seconds. ${ }^{15}$ The experimental conditions were randomized and balanced. All exercise sessions were carried out from 7 to 12 o'clock, with 48-hour interval between the sessions.

During each session, Tcore, skin temperature $\left(\mathrm{T}_{\text {skin }}\right)$ and $\mathrm{VO}_{2}$ were recorded every minute. Measurements of the Tcore were made by telemetry (ER-4000 energizer/receptor, Mini-Mitter Respironics, USA). $T_{\text {skin }}$ was measured using a thermometer (THR-140, Instrutherm Instruments, Brazil) connected to a thermocouple (S-09K, Instrutherm Instruments, Brazil) using an impermeable adhesive tape at approximately $20 \mathrm{~mm}$ from the lateral base of the tail. ${ }^{16} \mathrm{VO}_{2}\left(\mathrm{ml} . \mathrm{Kg}^{-0.75} \cdot \mathrm{min}^{-1}\right)$ was measured by an open-circuit indirect calorimetry system (Panlab, Harvard Apparatus, Spain). The temperature was maintained at $25^{\circ} \mathrm{C}$ throughout the exercise session.

\section{Calculations}

Work $(\mathrm{W})=$ body mass $(\mathrm{Kg}) \cdot$ force of gravity $\left(9.8 \mathrm{~m} / \mathrm{s}^{2}\right) \cdot$ TET (min).treadmill speed $\left(\mathrm{m} \cdot \mathrm{min}^{-1}\right) \cdot \cos \theta$ (treadmill slope). ${ }^{17}$ $\mathrm{ME}=\left(\mathrm{W} / \mathrm{energy}^{\mathrm{cost}}\right) \cdot 100$. $^{\mathrm{T}}$

The threshold for cutaneous heat loss was defined as the mean Tcore registered at the time when $T_{\text {skin }}$ significantly increased from the lowest measure registered during exercise. ${ }^{8}$

Heat loss sensitivity was calculated from the regression slope of Tcore and $T_{\text {skin }}$ during the first four minutes after the threshold was achieved. ${ }^{8}$ Heat accumulation $(\mathrm{HA})=(\Delta \mathrm{T}$ core $) \cdot$ body mass $(\mathrm{g}) \cdot \mathrm{h}$, where $\Delta \mathrm{Tcore}$ corresponded to variation of Tcore $\left(\mathrm{T}_{\text {final }}-T_{\text {initial }}\right)$, and $\mathrm{h}$ corresponds to specific heat of body tissues $\left(0.826\right.$ cal. $\left.\mathrm{g}^{-1} .{ }^{\circ} \mathrm{C}^{-1}\right) .{ }^{18} \mathrm{HA}$ was normalized by $100 \mathrm{~g}$ of body mass. The HA/W ratio $\left(\mathrm{cal}^{-\mathrm{j}^{-1}}\right.$ ) was considered an index of thermal efficiency.

\section{Statistical analysis}

Data normality was tested using the Shapiro-Wilk test. Normally distributed variables were expressed as mean \pm SD . Tcore, $\mathrm{T}_{\text {skin }}$ and $\mathrm{VO}_{2}$ were compared using the two-way ANOVA followed by post-hoc analysis with t-test (LSD, Least Significant Difference) or the Scott Knott test, as appropriate. TET, E, $\mathrm{ME}$, SBP, diastolic blood pressure (DBP), MBP and RHR were analyzed by two-way ANOVA followed by Tukey's post-hoc test. Paired t-test was used to assess the effects of low-intensity exercise on body mass, SBP, DBP, MBP and RHR. The level of significance was set at $5 \%$. All statistical analyses were performed using the Sisvar software, version 5.6 (Brazil).

\section{Results}

The effects of physical training on body mass, SBP, DBP, MBP, RHR and TET are described in Table 1. Body mass 
Table 1 - General characteristics of the animals studied; data expressed as mean \pm standard deviation

\begin{tabular}{|c|c|c|c|c|}
\hline Variable & C-WIS $(n=8)$ & T-WIS (n= 8) & C-SHR $(n=8)$ & T-SHR $(n=8)$ \\
\hline Initial BM (g) & $390.0 \pm 16.9$ & $356.6 \pm 23.7^{\text {\# }}$ & $258.6 \pm 14.7^{+}$ & $271.3 \pm 13.5^{+}$ \\
\hline Final BM (g) & $462.6 \pm 15.8$ * & $421.0 \pm 35.9$ *\# & $326.5 \pm 20.9^{*+}$ & $309.1 \pm 24.6^{*+}$ \\
\hline Initial SBP (mmHg) & $132.2 \pm 9.8$ & $123.7 \pm 7.6$ & $172.5 \pm 14.9^{+}$ & $189.7 \pm 9.6^{\#+}$ \\
\hline Final SBP $(\mathrm{mmHg})$ & $129.6 \pm 7.6$ & $127.8 \pm 8.7$ & $190.0 \pm 8.4^{*+}$ & $167.3 \pm 16.6^{* \#+}$ \\
\hline Initial DBP (mmHg) & $84.0 \pm 13.2$ & $90.0 \pm 13.2$ & $135.5 \pm 18.1^{+}$ & $143.3 \pm 17.8^{+}$ \\
\hline Final DBP $(\mathrm{mmHg})$ & $90.3 \pm 7.9$ & $98.5 \pm 14.1$ & $144.5 \pm 18.6^{+}$ & $117.3 \pm 28.0$ *\#+ \\
\hline Initial MBP (mmHg) & $100.3 \pm 10.7$ & $100.8 \pm 10.7$ & $147.6 \pm 16.1^{+}$ & $157.7 \pm 14.9^{\#+}$ \\
\hline Final MBP $(\mathrm{mmHg})$ & $104.0 \pm 7.3$ & $107.2 \pm 13.2$ & $158.6 \pm 12.1^{+}$ & $133.1 \pm 22.6$ *\#+ \\
\hline Initial RHR (bpm) & $338.7 \pm 19.5$ & $340.2 \pm 12.1$ & $374.2 \pm 11.0^{+}$ & $370.1 \pm 12.4^{+}$ \\
\hline Final RHR (bpm) & $337.5 \pm 10.7$ & $311.7 \pm 12.1$ * & $374.2 \pm 16.4^{+}$ & $365.7 \pm 18.6^{+}$ \\
\hline TET (min) & $21.9 \pm 1.9$ & $34.8 \pm 4.2 \#$ & $23.4 \pm 2.5$ & $28.4 \pm 3.6^{\#+}$ \\
\hline
\end{tabular}

C-WIS: control Wistar; T-WIS: trained Wistar, C-SHR: control SHR, T-SHR: trained SHR. BM: body mass; SBP: systolic blood pressure; DBP: diastolic blood pressure; MBP: mean blood pressure; RHR: resting heart rate; TET: total exercise time at week 8 of the incremental test. * $p<0.05$ : initial vs. final. \# $p<0.05$ : trained vs. controls within same lineage; $+p<0.05$ : WIS vs. SHR at the same training level

increased in all groups after 12 weeks of training. Lower body mass and higher SBP, DBP, MBP and RHR were observed in SHRs compared with Wistar rats. After the exercise program, RHR was significantly lower in Wistar rats, which was not observed in SHRs. Besides, the low-intensity physical training significantly reduced SBP (12\%), DBP (18\%) and MBP (12\%) in T-SHR, whereas the SBP increased in C-SHR after 12 weeks. The exercise training increased physical performance in both Wistar and SHR groups. Also, T-SHR showed lower physical capacity compared with T-WIS.

The effects of acute exercise (at $60 \%$ of RMS) on Tcore, $\mathrm{VO}_{2}$ and $\mathrm{T}_{\text {skin }}$ are described in Figure 1. Hypertension and physical training had no effect on Tcore during moderate exercise (Figure 1A). The T-WIS group showed higher $\mathrm{VO}_{2}$ (from 6 minutes to 16 minutes, and at the point of fatigue; Figure $1 \mathrm{~B}$ ) and $\mathrm{T}_{\text {skin }}$ (from 14 minutes to 18 minutes, and at the point of fatigue; Figure $1 \mathrm{C}$ ) compared with the WIS-C. The C-SHR group showed higher $\mathrm{T}_{\text {skin }}$ than the C-WIS (from 13 minutes to 17 minutes; Figure $1 \mathrm{C}$ ). Low-intensity training had no effect on $\mathrm{T}_{\text {skin }}$ or $\mathrm{VO}_{2}$ in SHR during moderate exercise. In addition, a lower $\mathrm{T}_{\text {skin }}$ was found in the T-SHR group at the point of fatigue compared with the T-WIS (Figure 1C).

Figure 2 shows the threshold and sensitivity of heat dissipation during acute exercise. These parameters did not change with hypertension or low-intensity physical training.

In addition, neither hypertension nor physical training affected W during acute exercise (Figure 3A). Hypertensive animals showed lower ME compared with normotensive animals, both in control and trained groups (Figure 3B). Also, physical training had no effect on ME in both Wistar and SHR (Figure 3B).

Results of HA and HA/W ratio are illustrated in Figure 4. The T-WIS group showed higher HA than the C-WIS (Figure 4A), and the T-SHR had lower HA compared with T-WIS (Figure 4A). However, when HA was corrected for W, no difference was found in the effects of $\mathrm{SAH}$ and physical training (Figure 4B).

\section{Discussion}

The present study aimed to evaluate the effects of low-intensity physical training on thermal balance in hypertensive rats subjected to an acute exercise program. We tested the hypothesis that low-intensity training could promote positive adaptations and ultimately reversal of the changes in the thermal balance of SHRs. For this purpose, we evaluated Tcore, heat production and heat dissipation in response to exercise. Altogether, our results showed that low-intensity physical training did not cause significant changes in the variables related to thermal balance, and thus, our hypothesis was rejected.

Thermal balance results from the relationship between heat production and dissipation, ${ }^{18}$ resulting in the Tcore regulation within satisfactory limits. During acute physical exercise, heat production occurs before heat dissipation, and consequently Tcore increases more rapidly than dissipation. ${ }^{19}$ This dynamics was observed in the present study (Figure 1) for the thermal balance variables in all experimental groups, i.e., for heat production $\left(\mathrm{VO}_{2}\right)$, heat dissipation $\left(\mathrm{T}_{\text {skin }}\right)$ and resulting outcome (Tcore). Throughout the exercise session, the Tcore threshold for heat dissipation is achieved and the thermoeffector response of heat dissipation occurs, measured by vasodilation in the tail skin. These adaptations allow achievement of thermal balance and regulation of Tcore within adequate limits, ${ }^{20}$ which was also observed in our study. An important adjustment, commonly reported in the literature, that confirms this pattern of response is the absence of vasodilation, and even occurrence of vasoconstriction, in the animals' tails in the beginning of exercise ${ }^{19}$ (Figure 1C).

Although recent studies of our group have shown that untrained SHR show disturbances in the regulation of body temperature during acute exercise, findings of the present study do not confirm the hypothesis that these thermal balance changes could be reversed by low-intensity aerobic physical training. In these previous studies, untrained SHR showed higher Tcore during constant-intensity acute exercise $(60 \%$ 
A

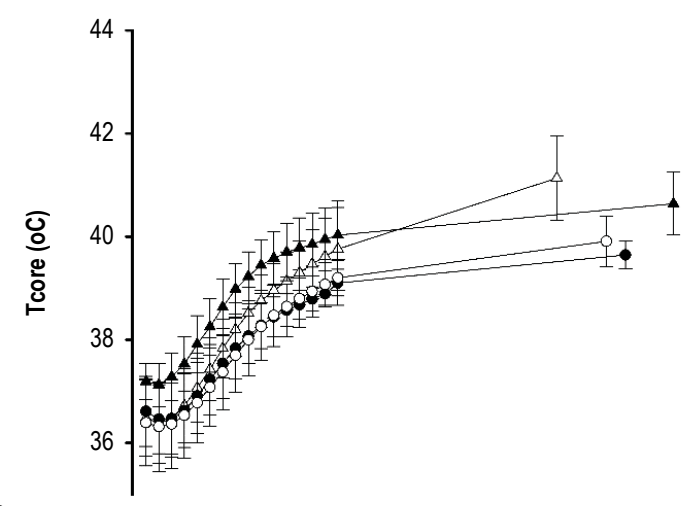

B
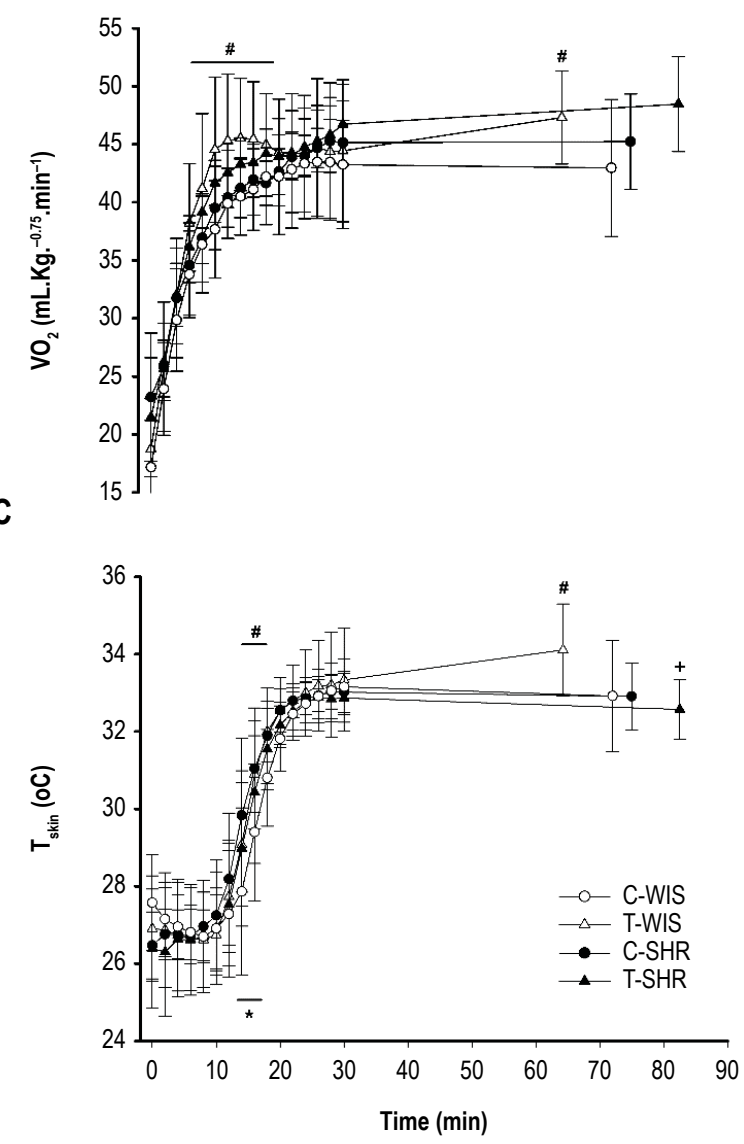

Figure 1 - Core temperature (Tcore, A), oxygen consumption $\left(\mathrm{VO}_{2}, B\right)$ and tail skin temperature $\left(T_{\text {skin }}, C\right)$ during acute exercise, until fatigue. Control Wistar (C-WIS), trained Wistar (T-WIS), control SHR (C-SHR), trained SHR (T-SHR). Data expressed as mean \pm SSD; ${ }^{*} p<0.05$ : C-SHR vs. C-WIS; \# $p<0.05$ : T-WIS vs. C-WIS; + $p<0.05$ : T-SHR vs. T-WIS.

MRS) associated with higher heat production and dissipation. ${ }^{7,8}$ It is worth pointing out that the age of the animals and the absolute running speed during the acute exercise protocol were different among these studies, which could explain this difference. Future studies should test other exercise intensities and duration, since the effects of training are known to be dependent on these variables. ${ }^{21}$
In the present study, the intensity of acute physical exercise (60\% of MRS) was established according to the American College of Sports Medicine recommendations. ${ }^{22}$ It is of note that, during the acute exercise session, although the animals were subjected to the same relative exercise intensity, the absolute speed was higher in trained animals. Gant et al. ${ }^{23}$ analyzed the relationship between Tcore and relative exercise 


\section{A}

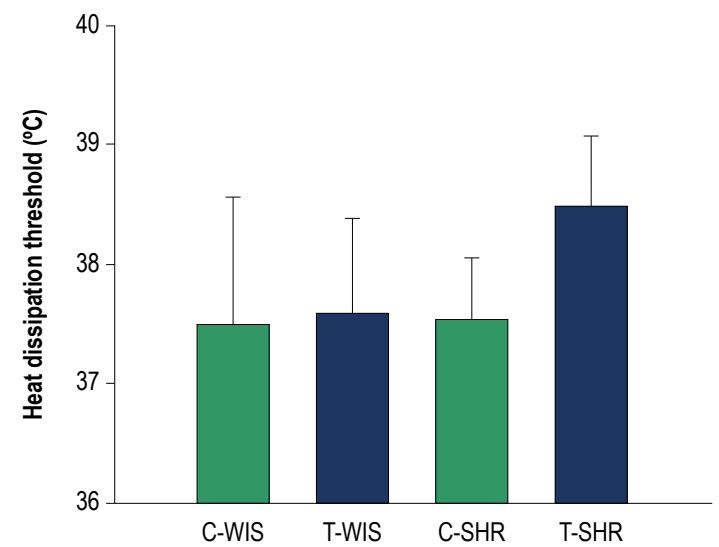

B

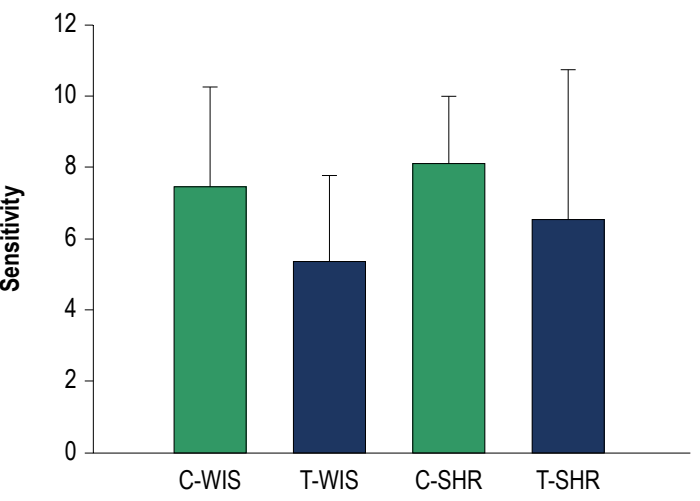

Figure 2 - Heat dissipation threshold $\left({ }^{\circ} \mathrm{C}\right)(\mathrm{A})$ and sensitivity $(B)$ during the acute physical exercise protocol. Data expressed as mean $\pm S D$. C-WIS: control Wistar, T-WIS: trained Wistar, C-SHR: control SHR, T-SHR: trained SHR.

intensity. Although the authors did not observe differences in Tcore between groups of animals with different $\mathrm{VO}_{2 \max }$ throughout one hour of exercise at $60 \%$ of $\mathrm{VO}_{2 \max }$ when subjected to exercise at similar absolute intensity, these groups showed different Tcore between them. These data suggest that the magnitude of hyperthermia may be associated with the absolute exercise load, regardless of the training status. In the present study, the T-WIS group showed greater heat production compared with the C-WIS group. This may be due to the higher intensity of exercise, which was counterbalanced by higher heat dissipation, resulting in comparable Tcore values in relation to the C-WIS group.

Low-intensity physical exercise increased physical capacity in SHR and reduced blood pressure, without promoting resting bradycardia. The mechanisms responsible for the reduction of blood pressure levels in hypertensive rats following aerobic physical training include structural, vascular and neurohumoral adaptations, such as reduction in sympathetic vasomotor activity, ${ }^{24,25}$ lower vascular reactivity, ${ }^{26}$ reduction in peripheral vascular resistance, ${ }^{27,28}$ reduction of oxidative stress, ${ }^{29}$ and changes in the endothelium-derived relaxing and contractile factors. ${ }^{30}$
Hypertensive animals showed lower ME compared with normotensive animals, as previously described. ${ }^{7}$ This could be explained, at least in part, by the higher proportion of type IIA fibers to type I fibers in the soleus muscle, as type I fibers are inherently more efficient than type IIA fibers. ${ }^{31}$ The physiological mechanisms responsible for the change of the muscle fiber profile may be associated with microcirculation rarefaction that precedes microvascular apoptosis, which would result in reduction of type I muscle fibers and augmented muscle anaerobiosis. ${ }^{31}$ However, the lower ME did not compromised work performance in the SHR group during acute physical exercise. Low-intensity exercise did not increase $M E$, neither in normotensive nor in hypertensive rats.

The present study has some limitations. It is possible that the difference in body mass between hypertensive and normotensive animals may have influenced the changes in Tcore induced by exercise, since the energy cost of running and heat dissipation from the skin depend on body mass. ${ }^{32}$ Nevertheless, this limitation is somewhat expected when both normotensive animals and SHR are studied, especially when they are matched by age. ${ }^{7,8,10}$ On the other hand, Drummond et 


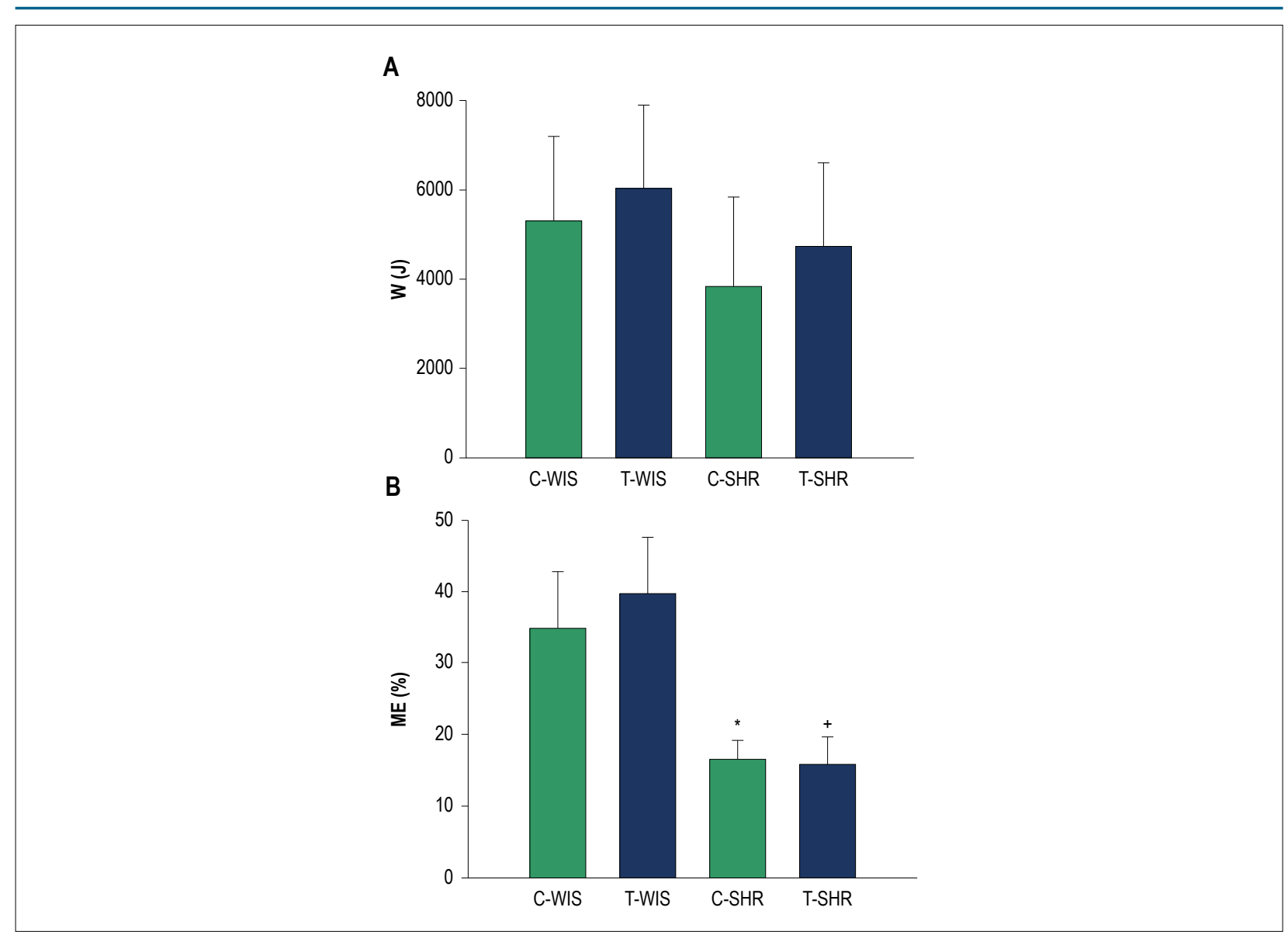

Figure 3 - Work (W, A) and mechanical efficiency (ME, B) during the acute physical exercise protocol. Data expressed as mean \pm SD; * $p<0.05: C-S H R$ vs. C-WIS; $+p<0.05$ : T-SHR vs. T-WIS. C-WIS: control Wistar, T-WIS: trained Wistar, C-SHR: control SHR, T-SHR: trained SHR.

al. ${ }^{8}$ demonstrated that differences in the thermoregulation between normotensive animals and SHR during acute exercise were not dependent on variations of body mass. These differences could also affect the ability of the animals to be trained, since they could be associated with differences in body composition, and consequently in differences in physical capacity. Finally, we cannot affirm that the results would have been the same if physical training had been started before a SBP higher than $150 \mathrm{mmHg}$ was achieved by the SHR, or if animals of different ages were studied.

\section{Conclusion}

Low-intensity physical training did not affect thermal balance in hypertensive rats subjected to an acute exercise protocol.

\section{Author contributions}

Conception and design of the research: Gomes LHLS, Natali AJ, Prímola-Gomes TN; Acquisition of data: Gomes LHLS, Rezende LMT, Prímola-Gomes TN; Analysis and interpretation of the data and critical revision of the manuscript for intellectual content: Gomes LHLS, Drummond LR, Campos HO, Rezende LMT, Carneiro-Júnior MA, Oliveira A, Natali AJ, Prímola-Gomes TN; Statistical analysis: Gomes LHLS, Drummond LR, Campos HO, Rezende LMT, Oliveira A,
Prímola-Gomes TN; Obtaining financing: Prímola-Gomes TN; Writing of the manuscript: Gomes LHLS, Drummond LR, Campos HO, Carneiro-Júnior MA, Prímola-Gomes TN.

\section{Potential Conflict of Interest}

No potential conflict of interest relevant to this article was reported.

\section{Sources of Funding}

This study was funded by FAPEMIG, CNPq and CAPES.

\section{Study Association}

This article is part of the thesis of master submitted by Luis Henrique Lobo Silame Gomes, from Universidade Federal de Viçosa.

\section{Ethics approval and consent to participate}

This study was approved by the Ethics Committee of the Universidade Federal de Viçosa under the protocol number 76/2014. All the procedures in this study were in accordance with the 1975 Helsinki Declaration, updated in 2013. Informed consent was obtained from all participants included in the study. 
A
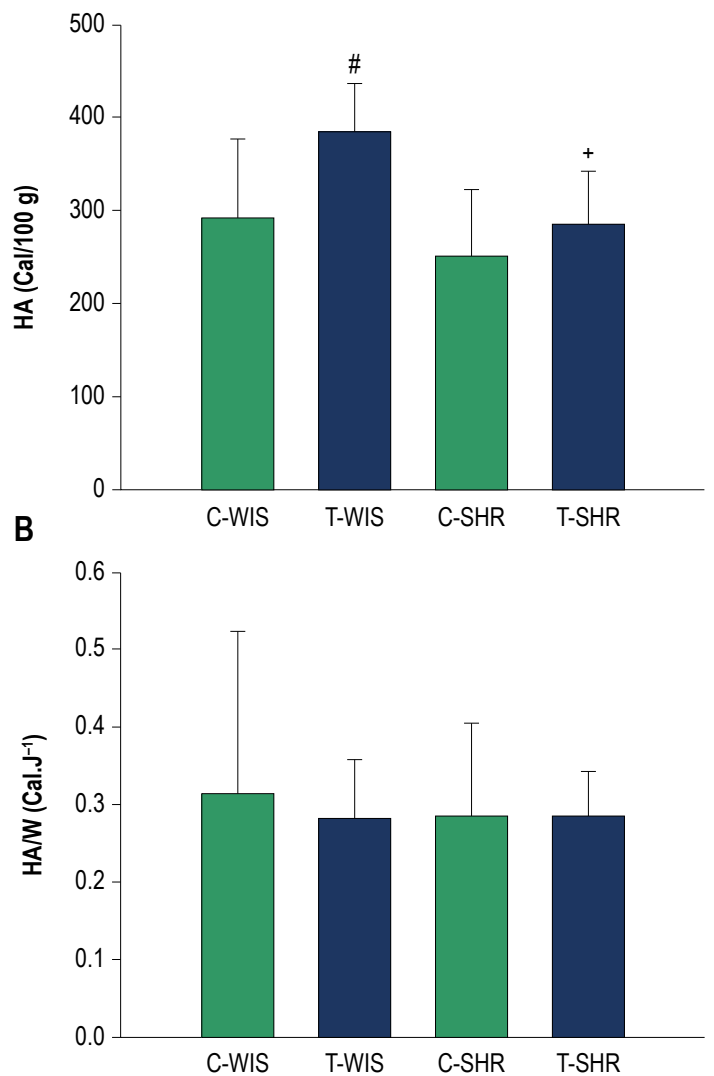

Figure 4 - Heat accumulation (HA, A) and heat accumulation/work ratio (HA/W, B). Data expressed as mean $\pm S D$; $\#<0.05: T-W I S$ vs. C-WIS. $+p<0.05: T$-SHR vs. T-WIS, C-WIS: control Wistar, T-WIS: trained Wistar, C-SHR: control SHR, T-SHR: trained SHR.

\section{References}

1. Webb P. The physiology of heat regulation. Am J Physiol. 1995;268(4 Pt 2):R838-50.

2. Gleeson M. Temperature regulation during exercise. Int J Sports Med. 1998;19(Suppl 2):S96-9.

3. Fuller A, Carter RN, Mitchell D. Brain and abdominal temperatures at fatigue in rats exercising in the heat. J Appl Physiol. 1998;84(3):877-83.

4. Chobanian AV, Bakris GL, Black HR, Cushman WC, Green LA, Izzo JL Jr et al. The Seventh Report of the Joint National Committee on Prevention, Detection, Evaluation, and Treatment of High Blood Pressure: the JNC 7 report. JAMA. 2003;289(19):2560-72.

5. Trippodo NC, Frohlich ED. Similarities of genetic (spontaneous) hypertension. Man and rat. Circ Res. 1981;48(3):309-19.

6. Cerbai E, Barbieri M, Li Q, Mugelli A. Ionic basis of action potential prolongation of hypertrophied cardiac myocytes isolated from hypertensive rats of different ages. Cardiovasc Res. 1994;28(8):1180-7.

7. Campos HO, Leite LH, Drummond LR, Cunha DN, Coimbra CC, Natali AJ, et al. Temperature control of hypertensive rats during moderate exercise in warm environment. J Sports Sci Med. 2014;13(3):695-701.

8. Drummond LR, Kunstetter AC, Vaz FF, Campos HO, Andrade AGP, Coimbra CC, et al. Brain temperature in Spontaneously Hypertensive Rats during physical exercise in temperate and warm environments. Plos One. 2016;11(5):e0155919.

9. Véras-Silva AS, Mattos KC, Gava NS, Brum PC, Negrão CE, Krieger EM. Low intensity exercise training decreases cardiac output and hypertension in spontaneously hypertensive rats. Am J Physiol. 1997;273(6 Pt 2):H2627-31.

10. Carneiro-Júnior MA, Quintão-Júnior JF, Drummond LR, Lavorato VN, Drummond FR, Cunha DN, et al. The benefits of endurance training in cardiomyocyte function in hypertensive rats are reversed within four weeks of detraining. J Mol Cell Cardiol. 2013 Apr;57:119-28.

11. Armitage P, Berry G. Statistical methods in medical research. Oxford: Blackwell; 1987.

12. Lavorato VN, Del Carlo RJ, Cunha DN, Okano BS, Belfort FG, De Freitas JS, et al. Mesenchymal stem cell therapy associated with endurance exercise training: effects on the structural and functional remodeling of infarcted rat hearts. J Mol Cell Cardiol. 2016 Jan; 90:111-9.

13. Prímola-Gomes TN, Pires W, Rodrigues LO, Coimbra CC, Marubayashi $U$, Lima NR. Activation of the central cholinergic pathway increases postexercise tail heat loss in rats. Neurosci Lett. 2007;413(1):1-5.

14. Pires W, Wanner SP, La Guardia RB, Rodrigues LO, Silveira AS, Coimbra CC, et al. Intracerebroventricular physostigmine enhances blood pressure and heat loss in running rats. J Physiol Pharmacol. 2007;58(1):3-17. 


\section{Original Article}

15. Soares DD, Lima NR, Coimbra CC, Marubayashi, U. Intracerebroventricular tryptophan increases heating and heat storage rate in exercising rats. Pharmacol Biochem Behav. 2004;78(2):255-61.

16. Young AA, Dawson NJ. Evidence for on-off control of heat dissipation from the tail of the rat. Can J Physiol Pharmacol. 1982;60(3):392-8.

17. Brooks GA, Donovan CM, White TP. Estimation of anaerobic energy production and efficiency in rats during exercise. J Appl Physiol Respir Environ Exerc Physiol. 1984;56(2):520-5.

18. Gordon CJ. Temperature regulation in laboratory rodents. Cambridge: Cambridge University Press; 1993.

19. Wanner SP, Primola-Gomes TN, Pires W, Guimaraes JB, Hudson AS, Kunstetter $\mathrm{AC}$, et al. Thermoregulatory responses in exercising rats: methodological aspects and relevance to human physiology. Temperature (Austin). 2015;2(4):457-75.

20. Romanovsky AA. Thermoregulation: some concepts have changed. Functional architecture of the thermoregulatory system. Am J Physiol Regul Integr Comp Physiol. 2007;292(1):R37-46.

21. Teixeira-Coelho F, Fonseca CG, Barbosa NHS, Vaz FF, Cordeiro LMS, Coimbra CC, et al. Effects of manipulating the duration and intensity of aerobic training sessions on the physical performance of rats. PLoS One. 2017;12(8):e0183763.

22. Pescatello LS, Franklin BA, Fagard R, Farquhar WB, Kelley GA, Ray CA, et al. American College of Sports Medicine position stand. Exercise and hypertension. Med Sci Sports Exerc. 2004;36(3):533-53.

23. Gant N, Williams C, King J, Hodge BJ. Thermoregulatory responses to exercise: relative versus absolute intensity. J Sports Sci. 2004;22(1112):1083-90.
24. Ceroni A, Chaar LJ, Bombein RL, Michelini LC. Chronic absence of baroreceptor inputs prevents training-induced cardiovascular adjustments in normotensive and spontaneously hypertensive rats. Exp Physiol. 2009;94(6):630-40.

25. Mueller PJ. Physical (in)activity-dependent alterations at the rostral ventrolateral medulla: influence on sympathetic nervous system regulation. Am J Physiol Regul Integr Comp Physiol. 2010;298(6):R1468-74.

26. Pasqualini L, Schillaci G, Innocente S, Pucci G, Coscia F, Siepi D, et al. Lifestyle intervention improves microvascular reactivity and increasesserum adiponectin in overweight hypertensive patients. Nutr Metab Cardiovasc Dis. 2010;20(2):87-92.

27. Melo RM, Martinho E, Michelini LC. Training-induced, pressure-lowering effect in SHR: wide effects on circulatory profile of exercised and nonexercised muscles. Hypertension. 2003;42(4):851-7.

28. Amaral SL, Michelini LC. Effect of gender on training-induced vascular remodeling in SHR. Braz J Med Biol Res. 2011;44(9):814-26.

29. Chaar LJ, Alves TP, Batista Junior AM, Michelini LC. Early Training-Induced Reduction of Angiotensinogen in Autonomic Areas-The Main Effect of Exercise on Brain Renin-Angiotensin System in Hypertensive Rats. PLoS One. 2015; 10(9): e0137395

30. Laughlin MH, Schrage WG, Mcallister RM, Garverick HA, Jones AW. Interaction of gender and exercise training: vasomotor reactivity of porcine skeletal muscle arteries. J Appl Physiol. 2001;90(1):216-27.

31. Damatto RL, Martinez PF, Lima AR, Cezar MD, Campos DH, Oliveira Jr SA, et al. Heart failure-induced skeletal myopathy in spontaneously hypertensive rats. Int J Cardiol. 2013;167(3):698-703.

32. Cramer MN, Jay O. Selecting the correct exercise intensity for unbiased comparisons of thermoregulatory responses between groups of different mass and surface area. J Appl Physiol. 2014:116(9);1123-32. 
